\title{
COMMENT
}

${ }^{\prime}$ Barcelona Institute for Global Health (ISGlobal), Hospital Clínic, University of Barcelona, Barcelona, Spain.

${ }^{2}$ EASL International Liver Foundation, Geneva, Switzerland.

${ }^{3}$ Department of Medicine, Humanitas Hospital, Rozzano, Italy.

${ }^{4}$ Clínica Universitária de Gastrenterologia, Laboratório de Nutrição, Faculdade de Medicina, Universidade de Lisboa, Lisboa, Portugal.

${ }^{5}$ Center for Systems and Community Design and NYU-CUNY Prevention Research Center, Graduate School of Public Health and Health Policy, City University of New York, New York, NY, USA.

${ }^{6}$ University of California Berkeley, School of Public Health, Forum for

Collaborative Research, Washington DC, DC, USA.

${ }^{7}$ Hepatitis Education Project, Seattle, WA, USA

${ }^{8}$ World Hepatitis Alliance, London, UK.

${ }^{9}$ Metabolic Liver Research Program, I. Department of Medicine, University Medical Center, Mainz, Germany.

${ }^{10}$ American Liver Foundation, New York, NY, USA

${ }^{11}$ Department of Medicine and Therapeutics, The Chinese University of Hong Kong, Hong Kong, Hong Kong.

${ }^{12}$ School of Public Health, University of Haifa,

Haifa, Israel.

${ }^{13}$ Department of Gastroenterology, Tel-Aviv Medical Center, Tel-Aviv, Israel.

凶e-mail: jeffrey.lazarus@ isglobal.org

\section{NAFLD - sounding the alarm on a silent epidemic}

Jeffrey V. Lazarus (10,2凶, Massimo Colombo ${ }^{2,3}$, Helena Cortez-Pinto $\mathbb{1}^{4}$, Terry T. -K. Huang ${ }^{5}$, Veronica Miller ${ }^{6}$, Michael Ninburg $\mathbb{1 0}^{7,8}$, Jörn M. Schattenberg ${ }^{9}{ }^{9}$, Lynn Seim ${ }^{10}$, Vincent W. S. Wong (1) ${ }^{11}$ and Shira Zelber-Sagi(i) ${ }^{12,13}$

On 'International NASH Day', we launch a call for the global health community to collaboratively shape and deliver a comprehensive, long-term public health agenda for NAFLD. A global multidisciplinary coalition is needed to guide our response to this increasingly prevalent, yet underaddressed disease.

We stand at a crossroads in how to address the public health challenge of nonalcoholic fatty liver disease (NAFLD). Our understanding of its natural history and basic biology has advanced substantially over the past decade, but there has been little progress in the public health response. While other non-communicable diseases (NCDs) have received increased attention, NAFLD has been absent from the public health discourse, including those discussions focused on NCDs. Moreover, in the absence of approved pharmacological treatments, little attention has been given to the clinical care pathways for patients with NAFLD.

In this Comment, published to mark 'International NASH Day' (12th June), we set forth what needs to be done by both liver health specialists and other stakeholders to advance the NAFLD global public health agenda and deliver a comprehensive response.

\section{NAFLD: a silent epidemic}

The first half of 2020 has brought the importance of health to prosperous and successful societies into sharp focus. The coronavirus disease 2019 (COVID-19) pandemic has prompted unprecedented policy action, which has affected daily life worldwide, with the express aim of saving lives and protecting the most vulnerable.

Rapid-onset crises such as SARS (severe acute respiratory syndrome) and COVID- $19^{1}$ have an unrivalled ability to capture the imagination and spur us into an immediate, emergency response. Yet most of the twentyfirst century's public health challenges - obesity, diabetes and hypertension, to name just three - are chronic and gradual in nature, making them almost imperceptible, so at best we feel ambivalent, at worst, blindly accepting.

NAFLD is the most prevalent liver disease in human history, with prevalence estimates indicating it affects almost two billion people globally ${ }^{2}$. Yet it is largely unknown to the general population, policymakers and even the global public health community. Despite the already staggering number, the NAFLD burden is expected to grow in the coming decades ${ }^{3}$, compromising individual health, burdening health-care systems and causing substantial economic and well-being losses ${ }^{4}$.

First identified in the 1980s, NAFLD is an umbrella term that describes a histological spectrum ranging from excess fat deposits in the liver (steatosis) to the more aggressive nonalcoholic steatohepatitis (NASH), which is characterized by hepatic inflammation (steatohepatitis) that prompts scarring of liver tissue (fibrosis $)^{5}$. Alongside the progressive liver damage, NAFLD is becoming an established risk factor for the leading causes of death and disability in the twenty-first century, namely cancer, cardiovascular disease and type 2 diabetes mellitus. Fortunately, in the past two decades, we have come to appreciate the complex relationships between NAFLD, obesity and many modifiable risk factors, such as lack of exercise, and have started to elucidate some of the genetic drivers of NAFLD ${ }^{6}$.

Despite these advances, even less attention has been paid to the prevention of liver disease through public health responses ${ }^{7}$, with NAFLD largely absent from the global public health and development agendas, such as the United Nations' Sustainable Development Goals ${ }^{8}$, nor is there yet any approved pharmacological treatment for NASH.

\section{A societal failure}

NCDs such as obesity, diabetes and NAFLD are often referred to as self-inflicted diseases, implying that personal behavioural choices are primary determinants of our chances of developing these conditions. This conveniently simplistic framing places the responsibility on the individual rather than acknowledging the influence that the obesogenic environment has on the choices of children and adults, affecting growth, development 
and health. This perception of personal blame leads to shame and stigmatization, compounds the burden on patients and reduces their motivation to seek help. However, this narrative is based on a false premise: that we have full agency over the choices we make irrespective of external forces. In fact, policies that prioritize profits over health have exacerbated social inequality and shaped all aspects of our environment, which, in turn, heavily influences our behaviour.

We have developed a NAFLD conceptual framework outlining how different factors contribute to the condition (FIG. 1). An individual's economic, political and social situation directly influences one's financial, physical and social capital. This influence, in turn, affects how the individual interacts with social systems, including those related to health, food, education, welfare and the built environment. Subsequently, these interactions influence an individual's behaviours regarding food, physical activity, work and the utilization of health-care and social services.

NAFLD thrives in communities that are designed around cars and not people, where healthy diets and access to health-care services are determined by socioeconomic status, and where commerce trumps public health ${ }^{9}$. NAFLD thus depends less on individual choices and more on socioeconomic and environmental conditions. Only by understanding and embracing this complex reality can we fully address the challenge of NAFLD.

\section{Connecting the dots}

It is clear that coordinated, collaborative action across sectors and disciplines is needed to achieve a society in which NAFLD is rare. The starting point should be to provide access to quality, affordable care to the hundreds of millions of people with NAFLD who now lack it.

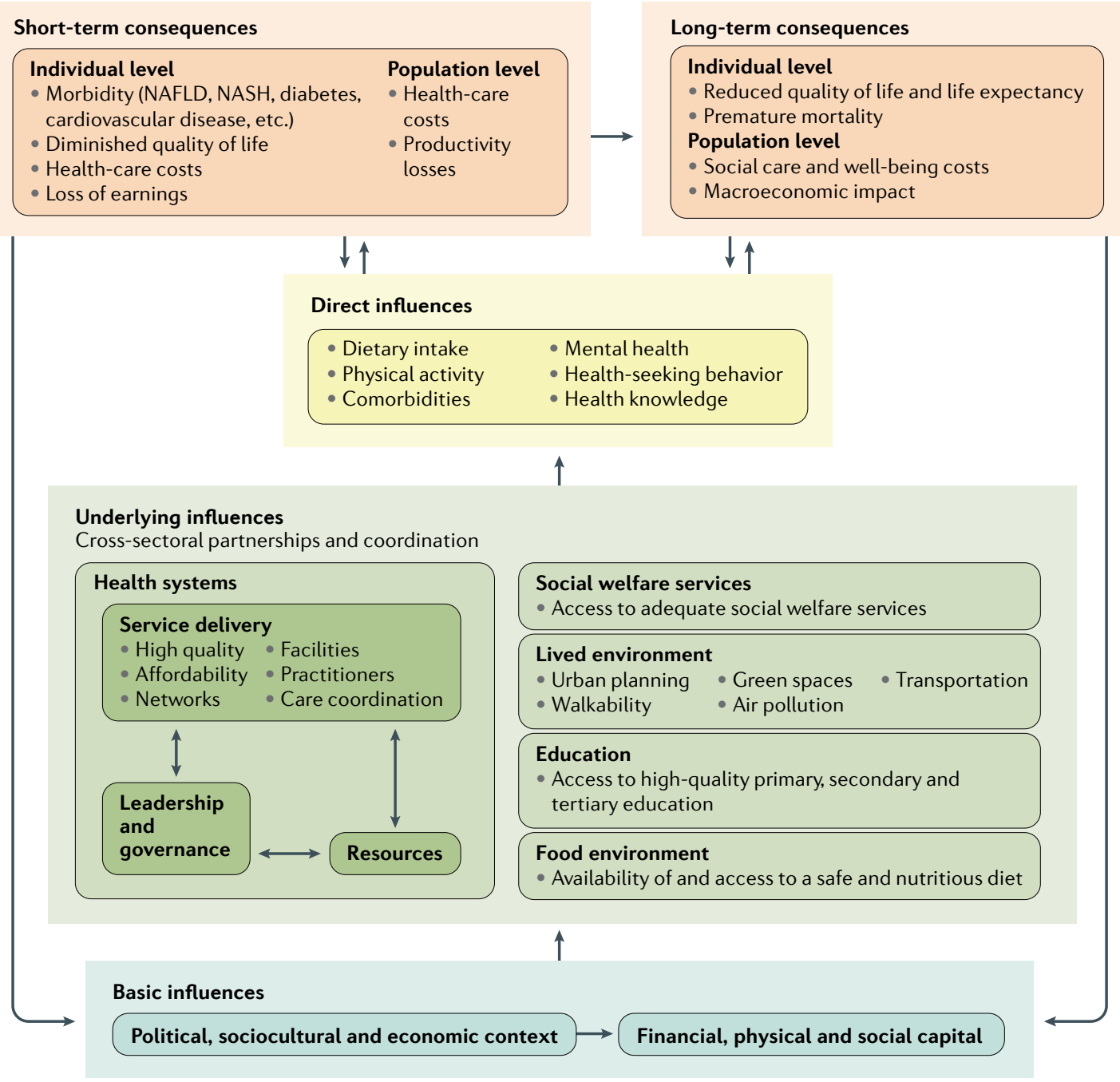

Fig. 1 | NAFLD conceptual model. The nonalcoholic fatty liver disease (NAFLD) conceptual framework outlines the factors influencing an individual's risk of developing NAFLD and the short-term and long-term consequences for individuals and populations. The bottom section (blue) reflects the political, economic and cultural context and an individual's financial, physical and social capital, which affect how they engage and interact with key social sectors and systems. An individual's interactions with these systems have a direct influence on the risk factors for developing NAFLD. The arrows highlight the main interactions between the three sections and the direction of the relationships. NASH, nonalcoholic steatohepatitis. 
We also need to improve our understanding of how to provide patient-centred care to people with NAFLD, with multidisciplinary models of care tailored to their place on the disease spectrum. To do so, the liver health community can draw on their experiences in improving care for people with viral hepatitis, such as focusing on the development of multidisciplinary models of care. The complex nature of NAFLD and its common comorbidities, including obesity and diabetes, calls for collaborating closely with those in other specializations, including endocrinologists and cardiologists, which is a particular challenge given the siloed way in which many health-care systems are designed. Concerted efforts are needed to overcome this health-care system barrier.

Although we in the liver health community should assume a central role in advocating for patients with NAFLD, we should seek to actively engage and collaborate with the broader NCD community to transform food systems and urban planning, and to create sustainable economies, which will be central to achieving a world without NAFLD. Our goal should be to fully integrate NAFLD into the NCD policy agenda, including key policy instruments addressing universal health coverage and the post- 2030 global health agenda. The health community, including researchers, clinicians and community advocates, have a responsibility to society to engage in and support measures that will deliver healthier environments and real-life impact for the majority of the population.

\section{A long and winding road}

NAFLD poses a classic challenge in contemporary health, one that will require the liver health community to embrace new, collaborative ways of working to deliver a holistic response. This process has already begun with a call from liver health clinicians for renaming NAFLD to include positive diagnostic criteria rather than having a diagnosis of exclusion; concretely they suggest a change to MAFLD (metabolic associated fatty liver disease $)^{10}$. For now, our contribution to the ongoing nomenclature debate is a simple one: avoid spelling out the acronym. Just think about how AIDS, MERS and SARS are used (Supplementary Box 1).

Ultimately, although progress is bound to be incremental, it is critical that we embark upon the proper trajectory now to lay the foundation for achieving our long-term goals. With that in mind, together with other key stakeholders, in late 2020 we are launching a global coalition to bring together actors from across a wide range of sectors and disciplines to support the response to this silent yet pernicious health challenge.

1. Zhang, C., Shi, L. \& Wang, F. S. Liver injury in COVID-19: management and challenges. Lancet Gastroenterol. Hepatol. https://doi.org/10.1016/s2468-1253(20)30057-1 (2020).

2. Younossi, Z. M. et al. Global epidemiology of nonalcoholic fatty liver disease-Meta-analytic assessment of prevalence, incidence, and outcomes. Hepatology 64, 73-84 (2016).

3. Estes, C. et al. Modelling NAFLD disease burden in China, France, Germany, Italy, Japan, Spain, United Kingdom, and United States for the period 2016-2030. J. Hepatol. 69, 896-904 (2018).

4. Schattenberg, J. et al. The economic burden of patients diagnosed with non-alcoholic steatohepatitis in France, Germany, Italy, Spain and the United Kingdom in 2018 (Poster) https://www. postersessiononline.eu/173580348_eu/congresos/NAFLD2019/ aula/-P02_2_NAFLD2019.pdf (2019).

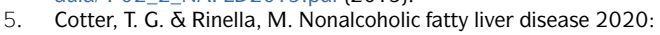 the state of the disease. Gastroenterology https://doi.org/10.1053/ j.gastro.2020.01.052 (2020).

6. Krawczyk, M., Liebe, R. \& Lammert, F. Toward genetic prediction of nonalcoholic fatty liver disease trajectories: PNPLA3 and beyond. Gastroenterology https://doi.org/10.1053/j.gastro.2020.01.053 (2020).

7. Lazarus, J. V. et al. A cross-sectional study of the public health response to non-alcoholic fatty liver disease in Europe. J. Hepatol. 72, 14-24 https://doi.org/10.1016/j.jhep.2019.08.027 (2020).

8. Lozano, R. et al. Measuring progress from 1990 to 2017 and projecting attainment to 2030 of the health-related Sustainable Development Goals for 195 countries and territories: a systematic analysis for the Global Burden of Disease Study 2017. Lancet 392, 2091-2138 (2018).

9. European Association for the Study of the Liver. Obesity is feeding the rise in non-alcoholic fatty liver disease (NAFLD) across Europe. EASL https://easl.eu/publication/policy-statement-obesity-is-feeding-therise-in-non-alcoholic-fatty-liver-disease-nafld-across-europe/ (2019).

10. Eslam, M., Sanyal, A. J. \& George, J. MAFLD: A consensus-driven proposed nomenclature for metabolic associated fatty liver disease. Gastroenterology https://doi.org/10.1053/j.gastro.2019.11.312 (2020).

\section{Competing interests}

The authors declare no completing interests.

Supplementary information

Supplementary information is available for this paper at https://doi.org/ 10.1038/s41575-020-0315-7. 